\title{
MODELIZACIÓN DE INTERFACES ADAPTATIVAS A PARTIR DE APRENDIZAJE DE PERFILES DE USUARIO WEB MEDIANTE GRAMÁTICAS PROBABILÍSTICAS
}

\author{
MODELING ADAPTIVE INTERFACES FROM LEARNING WEB \\ USER PROFILES USING PROBABILISTIC GRAMMARS
}

Augusto P. Cortez Vásquez' ${ }^{1}$, Ronald U. García Conde ${ }^{2}$

RECEPCIÓN: 20 DE NOVIEMBRE DE 2019

ACEPTACIÓN: 04 DE DICIEMBRE DE 2019

\section{RESUMEN}

En un sistema informático en la que interviene la maquina y el usuario se espera que estos respondan totalmente a lo esperado, o por lo menos lo suficiente para que la explotación del sistema pueda considerarse razonablemente aceptable. Lamentablemente esto no siempre ocurre. El problema de un diseño de interfaz de usuario inadecuado para aplicaciones informáticas se evidencia en que los usuarios no responden a lo que objetivamente se esperaba de ellos, ocasionando rechazos injustificados, deficiente utilización de los sistemas y, en algunos casos hasta errores y accidentes. El objetivo de la presente investigación es capturar las actividades de los usuarios durante su conexión y extraer patrones de comportamiento que permitan definir su perfil con el objetivo de diseñar interfaces adaptativas. Se utiliza las gramáticas probabilísticas libre de contexto que permite modelizar las sesiones de navegación de los usuarios. Se representa las sesiones web mediante grafos y gramáticas libres de contexto probabilísticas de tal forma que las sesiones que tengan mayor probabilidad son consideradas las más visitadas o más preferidas, por tanto, las más relevantes en relación a un tópico determinado. Se pretende desarrollar una herramienta para procesamiento de sesiones web obtenidas a partir de log de servidor representado mediante gramáticas probabilísticas libres de contexto.

\begin{abstract}
In a computer system involving the machine and the user, they are expected to fully respond to what is expected, or at least sufficiently so that the operation of the system can be considered reasonably acceptable. Unfortunately this doesn't always happen. The problem of an inadequate user interface design for computer applications is evidenced that users do not respond to what was objectively expected of them, causing unwarranted rejections, poor use of systems, and, in particular, some cases up to mistakes and accidents. The objective of this research is to capture the activities of users during their connection and extract patterns of behavior that allow to define their profile with the aim of designing adaptive interfaces. Context-free probabilistic grammars are used to model users' browsing sessions. Web sessions are represented by graphs and grammars free of probabilistic context in such a way that the sessions that are most likely are considered the most visited or preferred, therefore the most relevant in relation to a topic Determined. It is intended to develop a tool for processing web sessions obtained from server log represented by context-free probabilistic grammars.
\end{abstract}

Keywords: Interface; Adaptive interfaces; modeling adaptive interfaces; user profiles; probabilistic grammar; navigation patterns.

Palabras clave: interfaz; interfaces adaptativas; modelizacion deinterfacesadaptativas; perfiles deusuario; gramáticas probabilísticas; patrones de navegación.

\footnotetext{
1 Grupo de Investigación Biomedical, Universidad Nacional Mayor de San Marcos. Departamento de Ciencias de la Computación Perú. ORCID:0000-0002-5188-7962.

2 Universidad Tecnológica del Perú. Lima, Perú.

Autor para correspondencia: acortezv@unmsm.edu.pe,cortez_augusto@yahoo.fr
} 


\section{INTRODUCCION}

La abrumadora cantidad de información en la que nos sumergimos cuando navegamos en Internet nos causa una carga agobiante lo que en muchos casos desencadena en lo que los psicólogos denominan saturación cognitiva. La utilización efectiva de un sistema informático tiene estrecha relación con el diseńo de la interfaz de usuario, por lo que se hace evidente que no se debe considerar como un aspecto marginal. Pese a ello pese a ser un elemento esencial en el correcto uso de un sistema informático al momento de diseńar las interfaces de usuarios estos aspectos no son considerados. Por otro lado, debemos tener en consideración que no toso los usuarios respondemos de la misma forma en diferentes situaciones y contextos por lo que se hace necesario considerar el diseńo de interfaces adaptativas en función del perfil de cada usuario. En lo que respecta a nuestro aprendizaje, la Web ha propiciado replantarse totalmente nuestros procesos de aprendizaje y, por consiguiente, la accesibilidad y concepción de los recursos digitales educativos [16]. Cuando los usuarios navegan en el ciberespacio, se encuentran con un espacio amplio de información hipertextual, posibilitando un riesgo real de que se desorienten o tengan problemas para encontrar la información que necesitan. Basta que mirar a nuestro alrededor para atestiguar lo que el extraordinario poder de nuestra comprensión de la naturaleza nos ha ayudado a obtener. Cuando un usuario visita la web y quiere recuperar páginas en relación a un concepto, debe evitar muchas páginas irrelevantes, el objetivo es pues recuperar las paginas significativas, es decir aquellas que sean autoridad en el tópico. Frente a ello es imperativo reducir el espacio de búsqueda de los usuarios en función de su perfil cognitivo. Esto se podrá realmente si se tienen interfaces que se adapten al perfil del usuario en función de sus preferencias. Hay -grosso modo- dos conceptos relacionados en este artículo: identificar los perfiles de usuarios, para ello modelamos las sesiones de navegación mediante grafos y gramáticas probabilísticas de hipertexto, en segundo lugar, a partir de los perfiles especificar como se pueden diseñar páginas adaptativas a los usuarios en función de su perfil.

Para ello se utiliza un modelo simple de hipertextos representados mediante grafos, y se utiliza una representación de las sesiones de navegación de los usuarios inferidas de los archivos log como una gramática probabilística de hipertexto.

\section{OBJETIVOS}

\section{Objetivo general:}

- Obtener un instrumento para especificar interfaces adaptativas en función de los perfiles de usuarios web

\section{Objetivos específicos:}

- Aprender los patrones de navegación web mediante gramáticas probabilistas de hipertextos.

- Identificar los perfiles cognitivos de los usuarios en función de sus patrones de navegación

- Especificar interfaces adaptativas a partir de perfiles de usuarios

\section{MARCO CONCEPTUAL}

\subsection{Objetos de aprendizaje (OA)}

Diversos autores han coincidido en abordar una definición de Objeto de Aprendizaje como un conjunto de recursos producidos por una comunidad académica con el objetivo de intercambiarlos, integrarlos para producir a su vez nuevo aprendizaje. Sin embargo, es una tarea un tanto complicada, según señalan 
algunos autores menos optimistas, dado que esta debe soportarse en plataformas de tecnologías de información con el fin de que puedan ser reutilizables. Existe una amplia discusión respecto del término, dado a la permanente evolución de la tecnología y a las dificultades que se presentan en el proceso de adaptación a las necesidades educativas y tecnológicas. Los objetos de aprendizaje en el ámbito educativo se introducen sin considerar necesariamente a las Tecnologías de la Información y Comunicación (TIC), sin embargo, es a partir de éstas cuando cobra fuerza la idea de tener unidades de aprendizaje autocontenidas, interoperables -capacidad de integrarse en estructuras y plataformas diferentes-, reutilizables, durables y actualizables [16], [25]. Según Wiley "Los objetos de aprendizaje son los elementos de un nuevo tipo de instrucción basada en el computador y fundamentada en el paradigma computacional en la que el "objeto" es el centro de atención primordial. Es de vital importancia la creación de componentes (los "objetos") que pueden ser reutilizados en múltiples situaciones (contextos), en donde no solo interesa el lexema(texto), sino el sentido, el significado (es decir el concepto), como seńalan diversos autores, interesa no solo la sintaxis sino la semántica del lenguaje. Lo fundamental que encapsula el concepto de los objetos de aprendizaje: es que en su proceso de diseño se considera los aspectos de reutilización de componentes en contextos de estudio diversos y diferentes" [17], [25],

\subsection{Perfiles de usuario}

Numerosas son las nociones de perfil de usuario descritas por los autores, se ha destacado, sin embargo a menudo que una de las más importantes razones coincidentes para definir perfil de usuario es que es un conjunto de datos que permiten modelar los intereses y preferencias de un usuario en función de sus datos históricos almacenados en los archivos logs del sistema durante sus visitas en la web. Esta información almacenada permitirá detectar la forma como se comportan los usuarios en diferentes contextos, y a partir de estos construir patrones de comportamiento, lo que a su vez permitirá entender mejor las necesidades de este y así poder conocer sus intenciones [8] [22]. El principal objetivo de identificar los perfiles de usuario es describir las características, preferencias y necesidades de los usuarios, y en función de su perfil diseñar interfaces adaptativas con el propósito de diseñar mecanismos que permitan, recomendar objetos de aprendizaje, desde repositorios locales y remotos. El modelado de un perfil de usuario obedece a los criterios mostrados en la fig. 1. [12,15].

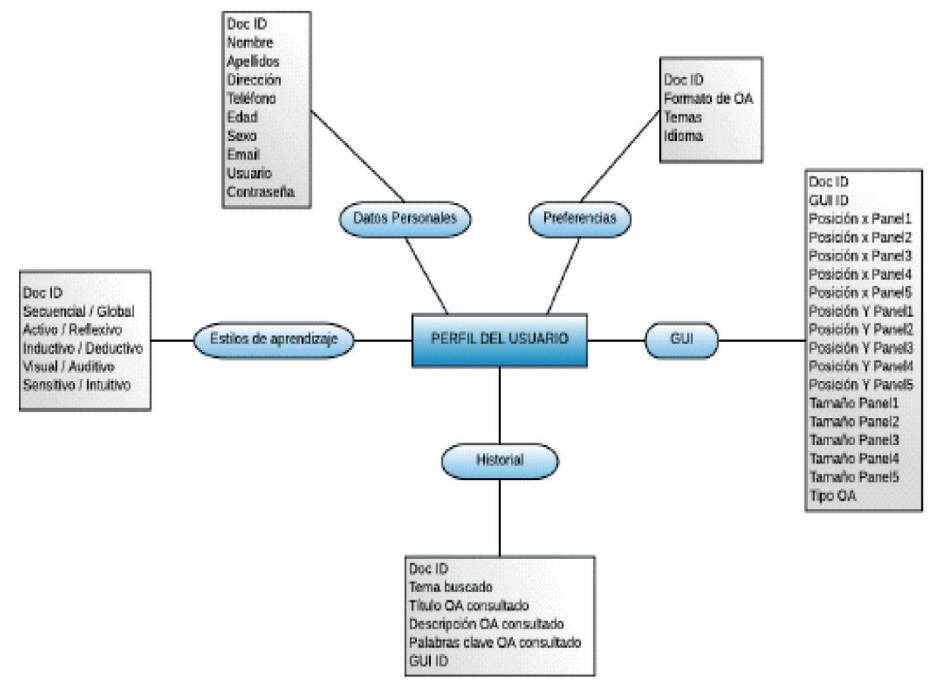

Fig. 1. Perfil de usuario (Quiroz, 2017) 
Aunque existen autores que confunden el concepto de "perfil de usuario" con el de "rol del usuario", nos circunscribiremos al concepto de perfil en función de los siguientes 5 elementos [22],

1. Datos personales: Permiten identificar unívocamente a cada usuario. Se consideran los siguientes atributos: (1) Documento único de identidad DNI , (2) Apellidos y Nombres, (3) Dirección, (4) Teléfono, (5) Edad, (6) Sexo, (7) Email, (8) Usuario y Contraseña, estos datos se almacenaran en el sistema permitiendo detectar patrones de comportamiento y en función de ello realizar adaptaciones a la GUI . Sin embargo, debe considerase que es un deber legal cuidar la vida privada de los usuarios

2. Estilo de aprendizaje: Todas las personas tienen formas diferentes de aprender, algunas prefieren aprendizaje textual, otros visual. En ese sentido es necesario recoger el tipo de cada usuario, sin embargo, se presentan ciertas dicotomías: (1) Secuencial / Global, (2) Activo / Reflexivo, (3) Inductivo / Deductivo, (4) Visual / Auditivo, (5) Sensitivo / Intuitivo, el SR tendrá en cuenta esta información para poder entregar los OAs adaptados a su estilo de aprendizaje.

3. Historial: Contendrá información de las búsquedas que ha realizado cada estudiante, los $\mathrm{OA}$ utilizados en cada tema de estudio y la distribución de la GUI en cada caso.

4. GUI: En este ítem se tiene identificada la distribución de los componentes de la interfaz gráfica utilizada por cada uno de los $\mathrm{OA}$, las que han sido seleccionada por el estudiante, esta información tendrá especial relevancia para el funcionamiento de la interfaz adaptativa.

5. Preferencias: Contendrá información relacionada con los temas de los OAs que ha seleccionado el usuario, los formatos que más utiliza (PDF, PNG, PPT, etc) y el/los idiomas de los OAs.

Cuando un usuario ingrese al sistema por primera vez, contestara un cuestionario a fin de identificar información importante y así poder empezar a alimentar el perfil del usuario con datos como: y (1) datos personales (2) el estilo de aprendizaje.

\subsection{Interfaces adaptativas ( IA)}

En forma muy general, una interfaz es un entorno de interacción persona-computador, la interfaz (o interfaz de usuario) es lo que permite la interacción entre persona y computador [21]. Las interfaces de usuario han evolucionado en las últimas décadas, desde interfaces eminentemente textuales a interfaces más complejas de tipo multimodal (integrando diversas formas de interacción tales como multimedia, táctil, vocal, etc.); lo anterior ha permitido mejorar la interacción hombre-máquina. El diseño de interface debe incluir aspectos relacionados a características básicas, el contexto y las preferencias cognitivas de los usuarios, que les permita de recomendar objetos de aprendizaje

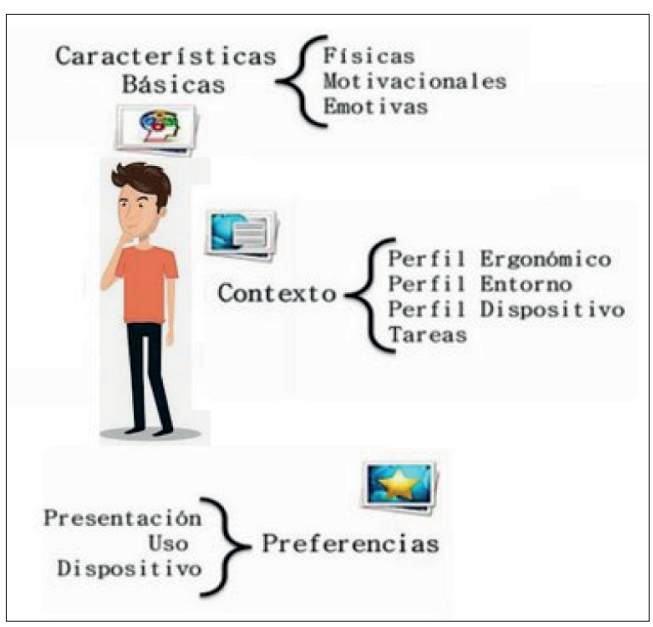

Fig 2. Consideraciones de la interface de usuario [22] (OAs) [14] [22], como se muestra en la fig. 2.

Diversos autores se refieren a las interfaces adaptativas como aquellas que se adaptan a las diferencias o cambios existentes entre los usuarios o algún contexto específico [23]. Como suele suceder con casi cualquier tecnología, la adaptación de los contenidos suele tener problemas que afectan a la 
interacción y la usabilidad, por un lado, y de manera directa por el otro, a la apreciación de utilidad de dicha tecnología por parte del usuario, lo que degenera en una pérdida de la motivación inicial y una bajada del grado de satisfacción de dichas experiencias docentes. El constante incremento de aplicaciones y formatos dificulta el trabajo con una única línea de productos o fabricantes, así como de contenidos, haciendo necesario el dominio e intercambio de formatos entre diferentes productos [13], [15].

Según López, [20], Uno de los ejemplos más utilizados de interfaces gráficas son las llamadas: WIMP (Windows Icons Menus Pointer), como la interfaz gráfica de Microsoft Windows o los escritorios para el sistema operativo Linux KDE o GNOME, que están basados en la interfaz gráfica presentada por Apple para sus MacIntosh. Existen distintas taxonomías que intentan clasificar la amplia variedad de posibles sistemas con algún grado de adaptación [20]. Sin embargo, a pesar de lo diverso y abundante, tradicionalmente se han considerado dos tipos de adaptación de la interfaz de usuario:

- Adaptabilidad: en este tipo de adaptaciones el usuario realiza la adaptación. Por lo tanto, es el usuario el que explícitamente adapta la interfaz de usuario para que se ajuste a sus gustos y características.

- Adaptividad: cuando se da este tipo de adaptación, el sistema es el actor responsable de realizar las acciones necesarias para realizar la adaptación.

\subsection{Lenguaje formal}

La expresión "lenguaje formal" se ha usado en varios sentidos y en diversos contextos. Sin embargo, diversos autores han coincidido en definir lenguaje formal como el conjunto de elementos que se rigen por reglas gramaticales rigurosas que no permiten ambigüedad en sus elementos, a diferencia de los lenguajes naturales que si lo permiten [18]. El lenguaje formal ha sido desarrollado por el hombre para expresar las situaciones que se dan en específico en cada área del conocimiento científico. Los lenguajes formales pueden ser utilizados para modelar una teoría de la mecánica, física, matemática, ingeniería eléctrica u otra en la que la característica fundamental es que no existe ambigüedad. Revisten especial importancia los lenguajes de programación de computadoras, y estas se definen considerando un conjunto de componentes léxicos, reglas gramaticales que definen la forma de las sentencias [2] [3] [6].

\subsection{Gramática}

Una gramática $\mathrm{G}$ es un modelo linguistico-matematico que hace referencia a la estructura de las sentencias de un lenguaje, es decir al orden sintáctico de los lexemas que componen las frases del lenguaje [1] [11]. Una gramática se define formalmente de la siguiente forma:

$\mathbf{G}=\left(\mathbf{V}_{\mathbf{t}}, \mathbf{V}_{\mathrm{N}}, \mathbf{P}, \mathbf{S}\right)$ donde:

$\mathbf{V}_{\mathrm{T}}$ : conjunto finito de símbolos terminales del lenguaje

$\mathbf{V}_{\mathrm{N}}$ : conjunto finito de símbolos no terminales

P : conjunto finito de reglas de producción

S : Símbolo distinguido o axioma inicial de la gramática

A partir del axioma $S$ se reconocerán las secuencias de $\mathrm{L}$ aplicando sucesivamente las reglas de producción.

\subsection{Gramática probabilista}

Una gramática libre de contexto probabilística (GLCP) es una gramática libre de contexto a la que se le ha añadido una probabilidad a cada regla de derivacikon[24]. La probabilidad de un análisis sintácti- 
co es el producto de las probabilidades de cada una de las reglas usadas en éste. De esta manera existen análisis que son más consistentes que otros. Nótese que las GLPC extienden las gramáticas libres de contextos incluyéndoles una función de probabilidad. [10][13].

Una PCFG se define entonces como una quíntupla $G=(V T, V 96 N, P, S, £]$ donde $£$ es una función para asignar probabilidades a cada regla en $\mathrm{P}$. La función $£$ expresa la probabilidad de que un no-terminal dado será expandido a la secuencia $\beta$. Una gramática probabilística tiene para cada regla P una probabilidad condicional, como se muestra en la Fig. 3:

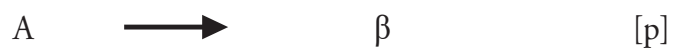

Asignar probabilidades a cada regla de producción

Luego de definir la gramática asignamos una probabilidad a cada regla de producción

\begin{tabular}{|c|c|c|}
\hline$S$ & NOMB VERB & {$[1.0]$} \\
\hline NOMB & ADJ NOMB & {$[0.3]$} \\
\hline NOMB & ADJ NOMB-SING & {$[0.7]$} \\
\hline VERB & VERB-SING ADVERV & {$[1.0]$} \\
\hline ADJ & $\mathrm{El}$ & {$[0.28]$} \\
\hline ADJ & $\mathrm{La}$ & {$[0.27]$} \\
\hline ADJ & Los & [0.12] \\
\hline ADJ & Las & {$[0.15]$} \\
\hline ADJ & Esos & {$[0.13]$} \\
\hline ADJ & Pequeño/traviesa & {$[0.7]$} \\
\hline NOMB-SING & niño & {$[0.32]$} \\
\hline NOMB-SING & niña & {$[0.37]$} \\
\hline NOMB-SING & señor & [0.19] \\
\hline NOMB-SING & señorita & {$[0.12]$} \\
\hline VERB-SING & escribe & {$[0.27]$} \\
\hline VERB-SING & corre & {$[0.16]$} \\
\hline VERB-SING & juega & {$[0.34]$} \\
\hline VERB-SING & salta & {$[0.23]$} \\
\hline ADVERV & rápidamente & {$[0.45]$} \\
\hline ADVERV & despacio & {$[0.28]$} \\
\hline ADVERV & frecuentemente & {$[0.27]$} \\
\hline
\end{tabular}

Fig.3. Gramática con sus probabilidades (propio)

\subsection{Gramática probabilista de hipertexto GPH}

La navegación hipertextual consiste en el proceso de seguir los enlaces de internet permitidos de una página a otra. Para navegar en la web desde una página $\mathrm{A}$ a otra $\mathrm{B}$, será menester que en la gramática exista una producción $\mathrm{A} \longrightarrow \mathrm{xB}$, que a permita transicionar desde la página $\mathrm{A}$ hacia la página $\mathrm{B}$, mediante el enlace $x$. Esta acción se materializará mediante un navegador WEB. Una GPH se define $\mathrm{G}=\left(\mathbf{V T}, \mathbf{V}_{\mathbf{N}}, \mathbf{P}, \mathbf{S}, £\right)$ gramática regular (definida por una expresión regular) con una relación uno-auno entre $\mathbf{V}_{\mathbf{N}}$ y $\mathbf{V}_{\mathbf{t}}$. [7] [13], 
Hernández señala [6] que las sesiones de navegación de los usuarios inferidas desde los archivos $\log$ pueden representarse como una gramática probabilista de hipertexto. Cada símbolo no terminal de $\mathrm{G}$ corresponde a una página visitada y cada regla de derivación corresponde a un enlace entre las páginas, Así la regla $\mathrm{A} \longrightarrow \mathrm{xB}$, significa la transición desde la página $\mathrm{A}$ hacia la página $\mathrm{B}$. Se advierte en ese sentido, que este método consiste en que las cadenas generadas por la gramática con mayor probabilidad corresponden a los caminos preferidos por los usuarios.

La probabilidad de una cadena de la gramática es el producto de las probabilidades de las producciones usadas en su derivación[13] [19]

\section{MÉTODO}

- Definición de la gramática: Se define la gramática G identificando los símbolos terminales, no terminales y las reglas de derivación.

- Definición de la gramática GPH: Se calcula la probabilidad de cada regla de producción asociada a la gramática.

- Definición de sesiones de navegación: A partir del log del servidor se construye un conjunto $P$ que contiene a las sesiones de navegación objeto de estudio.

- Construcción de grafo de sesiones: Se modela las sesiones mediante una estructura de grafo $G$.

- Especificación de perfil de usuario: Se identifican las áreas asociadas a cada página y estas se consignan en el perfil del usuario.

- Especificación de interface adaptativa: Definido el perfil del usuario, cuando este se identifica e ingresa a la web se diseńa en la interface adaptativa que incluye elementos asociadas al perfil del usuario.

\section{RESULTADOS Y DISCUSIÓN}

\subsection{Definición de GPH}

A partir del conjunto $P$ de sesiones de navegación obtenidas desde los archivos logs del servidor, se identifican las páginas involucradas, las que se representaran mediante símbolos no terminales de G.

$$
\mathbf{V}_{\mathrm{T}}:\left\{\mathrm{x}_{1}, \mathrm{x}_{2}, \mathrm{x}_{3}, \mathrm{x}_{4}, \mathrm{x}_{5}, \mathrm{x}_{6}, \mathrm{x}_{7}\right\} \quad \mathrm{V}_{\mathrm{N}}:\left\{\mathrm{A}_{1}, \mathrm{~A}_{2}, \mathrm{~A}_{3}, \mathrm{~A}_{4}, \mathrm{~A}_{5}, \mathrm{~A}_{6}, \mathrm{~A}_{7}\right\}
$$

Las reglas de producción se visualizan en el siguiente grafo (Fig. 4), en donde las aristas están etiquetadas con la probabilidad $P_{i j}$ de derivar $A_{i}$ a $A_{j}$

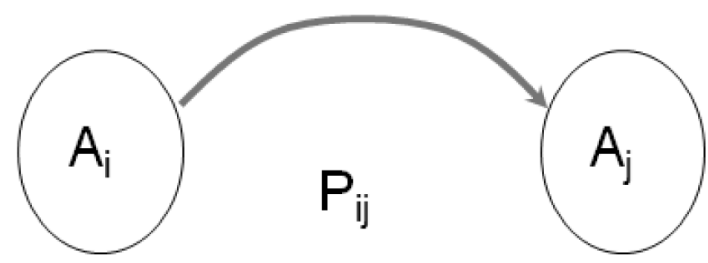

Fig.4. Modelo de transición de estados (propio) 
INDUSTRIAL

Una vez obtenidas las transiciones, se procederá a realizar el cálculo estadístico para asignar probabilidades[11]. Luego de determinar la cantidad de veces que se enlazan las páginas se calculan todas las probabilidades medias y condicionadas y el número de veces que se aplica una regla gramatical, como se muestra en la Fig. 5.

\begin{tabular}{|c|c|c|c|}
\hline Regla & $\begin{array}{c}\text { Ocurrencia } \\
\text { de } \alpha\end{array}$ & $\begin{array}{c}\text { Ocurrencia } \\
\text { de } \alpha \beta\end{array}$ & Probabil. \\
\hline $\mathrm{S} \longrightarrow \mathrm{x}_{1} \mathrm{~A}_{1}$ & 100 & 12 & 0.12 \\
\hline $\mathrm{S} \mathrm{x}_{2} \mathrm{~A}_{2}$ & 100 & 3 & 0.03 \\
\hline $\mathrm{S}_{\mathrm{x}_{3} \mathrm{~A}_{3}}$ & 100 & 8 & 0.08 \\
\hline $\mathrm{S}_{4} \mathrm{~A}_{4}$ & 100 & 9 & 0.09 \\
\hline $\mathrm{S}_{\mathrm{x}_{5}} \mathrm{~A}_{5}$ & 100 & 25 & 0.25 \\
\hline $\mathrm{S} \mathrm{x}_{6} \mathrm{~A}_{6}$ & 100 & 33 & 0.33 \\
\hline $\mathrm{S} \mathrm{x}_{7} \mathrm{~A}_{7}$ & 100 & 10 & 0.10 \\
\hline \multicolumn{4}{|l|}{ ……......... } \\
\hline$A_{6} x_{2} A_{7}$ & 50 & 16 & 0.32 \\
\hline$A_{6} x_{2} A_{7}$ & 50 & 34 & 0.68 \\
\hline $\mathrm{A}_{7} \mathrm{~F}$ & 15 & 15 & 1.00 \\
\hline
\end{tabular}

Fig. 5. Reglas de producción con probabilidades asignadas (propio)

Luego se amplía la gramática G a una gramática GPH.

Se distinguen las producciones en dos tipos:

- producciones de inicio, aquellas que comienzan con el axioma (S) y corresponde al inicio de una sesión,

- producciones transitivas aquellas que inician con un no terminal distinto a $S$, y corresponden a los enlaces entre páginas

El siguiente cuadro muestra la gramática con sus probabilidades:

\subsection{Grafo de sesiones}

Las reglas de producción se visualizan en el siguiente grafo (Fig. 6), en donde las aristas están etiquetadas con la probabilidad $P_{i j}$ de derivar $A_{i}$ a $A_{j}$

\begin{tabular}{|l|l|}
\hline 1) $\mathrm{S}_{\mathrm{a}_{1}} \mathrm{~A}_{1}(0.12)$ & 14) $\mathrm{A}_{2} \mathrm{a}_{5} \mathrm{~A}_{7}(0.32)$ \\
\hline 2) $\mathrm{S}_{2} \mathrm{~A}_{2}(0.03)$ & $15) \mathrm{A}_{4} \mathrm{a}_{5} \mathrm{~A}_{5}(0.26)$ \\
\hline 3) $\mathrm{S}_{3} \mathrm{a}_{3}(0.08)$ & $16) \mathrm{A}_{3} \mathrm{a}_{2} \mathrm{~A}_{4}(0.63)$ \\
\hline 4) $\mathrm{S}_{4} \mathrm{~A}_{4}(0.09)$ & $17) \mathrm{A}_{3} \mathrm{a}_{5} \mathrm{~A}_{6}(0.37)$ \\
\hline 5) $\mathrm{S}_{5} \mathrm{~A}_{5}(0.25)$ & $18) \mathrm{A}_{5} \mathrm{a}_{3} \mathrm{~A}_{6}(0.23)$ \\
\hline 6) $\mathrm{S}_{6} \mathrm{~A}_{6}(0.33)$ & 19) $\mathrm{A}_{5} \mathrm{a}_{2} \mathrm{~A}_{1}(0.30)$ \\
\hline 7) $\mathrm{S}_{7} \mathrm{~A}_{7}(0.10)$ & 20) $\mathrm{A}_{6} \mathrm{a}_{2} \mathrm{~A}_{7}(0.32)$ \\
\hline 8) $\mathrm{A}_{1} \mathrm{a}_{2} \mathrm{~A}_{3}(0.35)$ & 20) $\mathrm{A}_{1} \mathrm{~F}(0.30)$ \\
\hline 9) $\mathrm{A}_{1} \mathrm{a}_{4} \mathrm{~A}_{4}(0.12)$ & 21) $\mathrm{A}_{4} \mathrm{~F}(0.57)$ \\
\hline 10$) \mathrm{A}_{1} \mathrm{a}_{3} \mathrm{~A}_{7}(0.23)$ & 22) $\mathrm{A}_{5} \mathrm{~F}(0.47)$ \\
\hline 11) $\mathrm{A}_{4} \mathrm{a}_{2} \mathrm{~A}_{6}(0.17)$ & 23) $\mathrm{A}_{6} \mathrm{~F}(0.68)$ \\
\hline 12) $\mathrm{A}_{2} \mathrm{a}_{2} \mathrm{~A}_{3}(0.23)$ & 24) $\mathrm{A}_{7} \mathrm{~F}(0.10)$ \\
\hline 13) $\mathrm{A}_{6} \mathrm{a}_{4} \mathrm{~A}_{2}(0.45)$ & \\
\hline
\end{tabular}




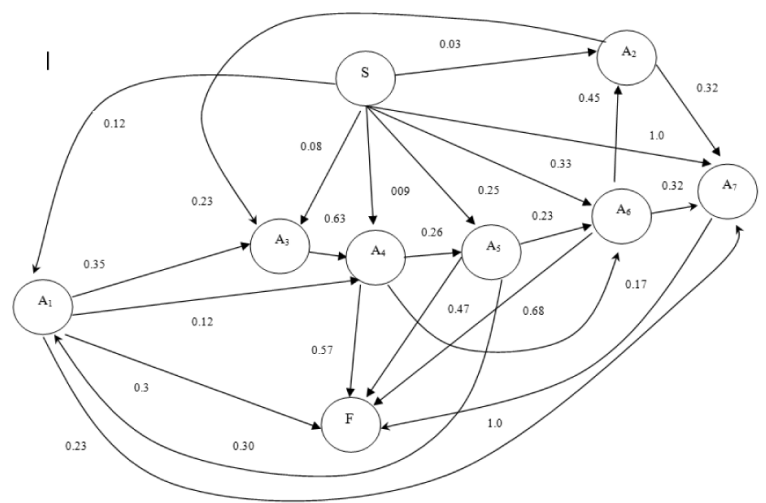

Fig. 6. Grafo de sesiones (propio)

\subsection{Determinación de probabilidades de sesiones}

En una sesión dos tipos de producciones: producciones de inicio, aquellas que inician con el axioma (S) y corresponde al inicio de una sesión, mientras que el resto, las que inician con un no terminal distinto a S, se denominan producciones transitivas y corresponden a los enlaces entre paginas [8] [13].

A partir de la gramática se pueden obtener cadenas, que representan a las sesiones de navegación de los usuarios, se trata de pues de realizar un cálculo estadístico sobre una colección de sesiones de navegación que permita obtener el número de veces que una página aparece como página inicial, el número de veces que aparece como página final y el número de veces que no es ni página inicial ni final [19]. A partir de esta estadística se obtendrá un patrón.

\begin{tabular}{|l|l|}
\hline ID & Sesión \\
\hline 1 & $\begin{array}{l}\mathrm{A}_{1} \rightarrow \mathrm{A}_{3} \rightarrow \mathrm{A}_{4} \rightarrow \mathrm{A}_{5} \rightarrow \mathrm{A}_{6} \rightarrow \\
\mathrm{A}_{7}\end{array}$ \\
\hline 2 & $\mathrm{~A}_{1} \rightarrow \mathrm{A}_{7}$ \\
\hline 3 & $\mathrm{~A}_{1} \rightarrow \mathrm{A}_{4} \rightarrow \mathrm{A}_{5} \rightarrow \mathrm{A}_{6}$ \\
\hline 4 & $\mathrm{~A}_{3} \rightarrow \mathrm{A}_{4} \mathrm{~A}_{6}$ \\
\hline 5 & $\mathrm{~A}_{4} \rightarrow \mathrm{A}_{5} \mathrm{~A}_{6} \mathrm{~A}_{2} \rightarrow \mathrm{A}_{7}$ \\
\hline 6 & $\mathrm{~A}_{1} \rightarrow \mathrm{A}_{4} \rightarrow \mathrm{A}_{5} \rightarrow \mathrm{A}_{1}$ \\
\hline 7 & $\begin{array}{l}\mathrm{A}_{5} \rightarrow \mathrm{A}_{6} \rightarrow \mathrm{A}_{2} \rightarrow \mathrm{A}_{3} \rightarrow \mathrm{A}_{4} \rightarrow \\
\mathrm{A}_{5}\end{array}$ \\
\hline 8 & $\begin{array}{l}\mathrm{A}_{3} \rightarrow \mathrm{A}_{4} \rightarrow \mathrm{A}_{5} \rightarrow \mathrm{A}_{1} \rightarrow \mathrm{A}_{4} \rightarrow \\
\mathrm{A}_{6}\end{array}$ \\
\hline
\end{tabular}

Sea

$S_{\mathrm{i}}$ : $\quad$ una sesión del conjunto $P$

$\mathrm{A}_{\mathrm{i}}$ : página involucrada en una sesión $\mathrm{Si}$

$\mathrm{r}_{\mathrm{i}}$ : número de veces que una página Ai fue requerida en las sesiones de $P$

$\mathrm{p}_{\mathrm{i}}$ : número de veces que una página Ai fue el primer estado en una sesión Si de $P$.

$\mathbf{u}_{\mathrm{i}}$ : número de veces que una página i fue el último estado en una sesión Si de $P$.

$\mathbf{t}_{\mathrm{ij}}$ : número de veces que una subsecuencia de dos páginas aparece en la sesión (página i y j) de $P$

$\boldsymbol{\alpha}>0$ : se pueden generar cadenas desde cualquier estado 
$\boldsymbol{\alpha}=0$ : solo los estados que fueron primeros en las sesiones actuales tienen probabilidad mayor que cero de ser una producción de inicio

$\boldsymbol{\alpha}>0$ : significa que se pueden generar cadenas desde cualquier estado

$\boldsymbol{\alpha}=1$ : significa que la probabilidad de una producción de inicio es directamente proporcional a la cantidad de veces que el correspondiente estado fue visitado. De o anterior se deduce que el nodo destino de una producción con más alta probabilidad (Fig. 7) corresponde al estado que fue visitado más a menudo, como se aprecia en la Fig. 8.

$\mathrm{N}: \mathrm{N} \geq 1$ : indica la cantidad de memoria del usuario cuando este navega por la red, es decir el número de URL anteriores que dependerán de la elección del siguiente URL

Si $\mathrm{N}=1$ : implica lo que se conoce como una cadena de markov, que es un tipo especial de proceso estocástico discreto en el que la probabilidad de que ocurra un evento depende del $\mathrm{P}(\mathrm{S} \rightarrow$ alAl $)=\frac{u . N-V-A_{1}+}{\text { N-T_V }} \frac{u . . \text { N-I-A }}{\text { N-T-I }}$ característica de falta de memoria recibe el nombre de

Si $\mathrm{N}=1$ y $\alpha=0.5$

donde:

$\mathbf{N}-\mathbf{V}-\mathbf{A}_{1}$ : número de visitas a $\mathrm{A} 1=6$

N-I-A $\mathbf{A}_{1}$ : número de inicios de $\mathrm{A} 1=4$

$\mathrm{P}(\mathrm{S} \rightarrow \mathrm{alAl})=\frac{0.5 \times 6+0.5 \times 4}{36} \frac{}{8}=0.33$

\begin{tabular}{|c|c|c|c|c|c|c|c|}
\hline PRD & Ucnow & & & TNNA & $\mathrm{MSA}_{4}$ & T-N-S & $P(p)$ \\
\hline$S \rightarrow$ & a1A1 & 0.5 & 6 & 36 & 4 & 8 & 0.33333333 \\
\hline $5 \rightarrow$ & $a_{4}, A 2$ & 0.5 & 2 & 36 & 0 & 8 & 0.02777778 \\
\hline $5 \rightarrow$ & a3A3 & 0.5 & 4 & 36 & 2 & 8 & 0.18055556 \\
\hline $5 \rightarrow$ & 24,4 & 0.5 & 7 & 36 & 1 & 8 & 0.15972222 \\
\hline $5 \rightarrow 5$ & a5A5 & 0.5 & 6 & 36 & 1 & 8 & 0.14583333 \\
\hline $5 \rightarrow$ & 2646 & 0.5 & 6 & 36 & 0 & 8 & 0.08333333 \\
\hline $5 \rightarrow$ & a7aj & 0.5 & 2 & 35 & 0 & 8 & 0.0277778 \\
\hline
\end{tabular}

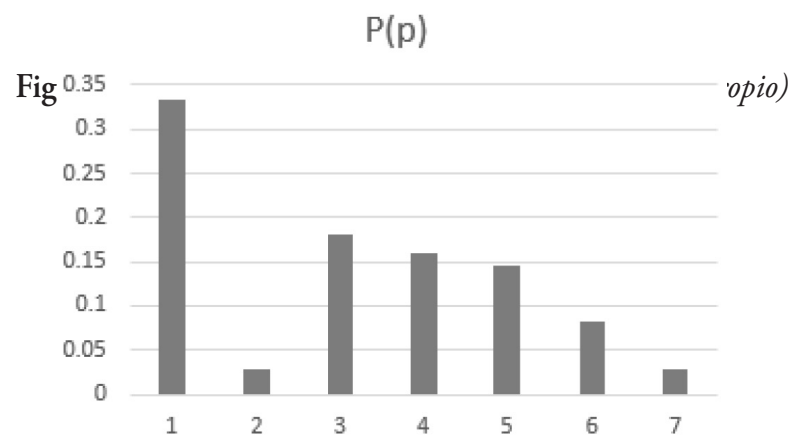


Fig. 8. Cuadro de producciones con sus probabilidades (propio)

\subsection{Identificación de perfil de usuarios}

A partir de los patrones de navegación se identifican las páginas más visitadas por los usuarios, que identifican sus preferencias

\begin{tabular}{|l|l|}
\hline Id sesión & Identificador de la sesión \\
\hline Id usuario & Identificador del usuario que inicia la sesión \\
\hline Ip & Ip del usuario que inicia la sesión \\
\hline Hora inicio & Fecha y hora que inicia la sesión \\
\hline Hora fin & Fecha y hora que finaliza la sesión \\
\hline NPV & Número de páginas accedidas en el sitio web \\
\hline NS & Número total de peticiones realizadas durante la sesión \\
\hline BD & Total de Bytes transferidos durante la sesión \\
\hline
\end{tabular}

Especificación de perfil de usuarios

Se crean cuatro vectores en donde se almacenan:

- Datos personales. Apellidos y nombres, correos, edad, sexo, teléfono

- Preferencias cognitivas. Ese vector se actualiza dinámicamente en función de las páginas visitadas: Páginas visitadas, temas, idiomas.

- Historial: Ese vector se almacenan los objetos de aprendizajes, sus palabras claves.

- Estilo de aprendizaje: Tipo de aprendizaje (Secuencial/global, Inductivo/deductivo, sensitivo/intuitivo).

\subsection{Especificación de interfaces adaptativas}

La especificación de la IA

Precondición: Interfaz actual, vectores

Adaptación Interfaz()

Postcondición: Interfaz adaptada al perfil

Adaptacion Interfaz() es el módulo encargado de efectivizar las adaptaciones de la interfaz (cambio de posición de componentes, iconos, botones, barrar, tamańos de los componentes, despliegue de OA recomendados). La adaptación se efectivizará, en función de los datos almacenados en el perfil de cada usuario.

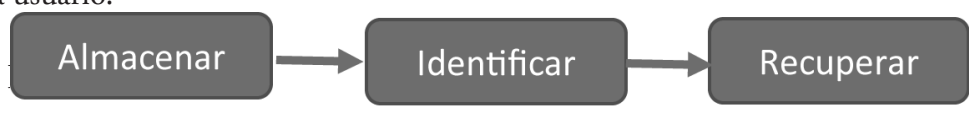

Estos tres mecanismos deben proveerse con carácter de

Inmediatez $\quad:$ obtener al momento los objetos que se necesitan

Flexibilidad : de fácil adaptación al usuario 
Personalización $\quad:$ ofrece rutas de aprendizaje alternativos

Elementos estructurales : Es el entorno material en que se produce la interacción entre el

Considera aspectos en tres niveles: usuario y el sistema.

1 Nivel físico: antropométricos y fisiológicos : se refiere a la adecuación del hardware a la conducta motora del usuario.

2 Nivel cognitivo: adecuación de la comunicación software/ mente En este nivel se considera:

a) Plano sintáctico/estructural: se refiere a las unidades de información; símbolos y palabras(lexemas) y las reglas que permiten construir estructuras (frases)

b) Plano semántico/constructivo: Se refiere a las reglas o criterios con los que se asigna significado a la información que se ofrece al usuario, con lo que se relaciona directamente con los procesos cognitivos de comprensión semántica(conceptos).

c) Plano pragmático dinámico: considera la forma en que se diseńa la navegación y esta relacionado con los procesos de toma de decisión

3 Nivel funcional: adecuación de las herramientas a los objetivos del usuario. El diseño de la interfaz debe servir para que el usuario se sienta cómodo y pueda utilizar la lo más fácil posible.

La interfaz está compuesta de paneles que permitirán al usuario

- Panel de Menú: En este panel se agrupan varias opciones para realizar algunas tareas como (desplegar los objetos de aprendizaje, Buscar OA, cambiar un OA etc.).

- Panel de búsqueda: La función de este panel es realizar búsquedas simples de OAs. Los OAs deben ser considerados como pequeńas piezas instruccionales que permitirán ensamblar para construir nuevos objetos.

- Panel de contenido: Este panel está enfocado en mostrar los OA que el usuario ha seleccionado. Con la finalidad que pueda reusar, reutilizar y ensamblar nuevos objetos

- Panel de repositorios: La función de este panel es permitir un acceso rápido a diferentes repositorios, permitiendo a los usuarios acceso a otros OA que no existen en el sistema.

El diseño de la interfaz adaptativa de usuario consiste en la articulación de los elementos antes seńalados en un "todo coherente" y no en partes aisladas o separadas

\section{CONCLUSIONES}

En la presente investigación se pone de relieve la importancia de las gramáticas como instrumento para aprender los patrones de navegación de los usuarios web.

A partir de los patrones de navegación del usuario se identifican los perfiles de los usuarios.

A partir de los perfiles de los usuarios se modelos las interfaces adaptativas.

El diseño de la interfaz adaptativa de usuario consiste en la articulación de los elementos antes señalados en un "todo coherente" y no en partes aisladas o separadas. 


\section{REFERENCIAS}

\section{Libros}

[1] A. Aho (2016). Compiladores, principios, técnicas y herramientas. Edit. DF Addison-Wesley, Wilmington-Delaware EUA. México.

[2] J. Brookshear (1993). Teoría de la computación. Addisson Wesley Iberoamericana Wilmington Delaware. México DF.

[3] J. Brookshear (2014). Computer Science: An Overview. México DF Pearson.

[4] A. Cortez (2014). Lenguajes y traductores. Edit. UCSS. Lima, Perú.

[5] J. Hernández (2008). Introducción a la minería de datos. Madrid. España. Pearson Prentice Hall España.

[6] J. Hopcroft (1993). Ullman "Introducción a la teoría de autómatas". Edit. CECSA. México DF.

[7] J. Palma (2008). Inteligencia artificial. Edit. MacGraw Hill. México.

[8] S. Russell (2013). Inteligencia artificial. Un enfoque moderno. Edit Pearson. México DF.

[9] L. Joyanes (2013). Big Data. Análisis de grandes volúmenes de datos en organizaciones. México DF.

\section{Artículos}

[10] A. Cortez (2009). "Procesamiento del lenguaje natural". Revista RISI vol 6. № 2, pp. 45-54, 2009. http://sisbib.unmsm.edu.pe/BibVirtual/Publicaciones/risi/2009_n2/v6n2/a06v6n2.pdf

[11] A. Cortez A. (2013). Gramáticas probabilistas, Revista Algorithmic 4(1), Pg 9-16 ISSN 22203982 Lima Perú

[12] F. Iriarte, F. (2005). Patrones de navegación hipertextual en usuarios inexpertos de sexto grado Fernando Iriarte Díaz Granados. Revista del Instituto de Estudios Superiores en Educación Universidad del Norte 6(6) ISSN 1657-2416. Recuperado:file://C:/Users/Cortez/AppData/ Local/Temp/Dialnet-PatronesDeNavegacionHipertextualEnUsuariosInexpert-2503227.pdf

[13] A. Cortez (2013). Sistema de Aprendizaje de Patrones de Navegación Web Mediante Gramáticas Probabilísticas de Hipertexto. Revista INGE CUC 11(1) pp. 72-78, 2013. file://C:/Users/ Cortez/AppData/Local/Temp/Dialnet-SistemaDeAprendizajeDePatronesDeNavegacionWebMe dia-5178333.pdf

[14] A. Cortez (2015). "Sistemas de Aprendizaje basados en Entornos Hipermedia adaptativos". Revista Theorema Vol. 2 , N. ${ }^{\circ} 3$, pp.155-164. https://revistasinvestigacion.unmsm.edu.pe/index. $\mathrm{php} /$ Theo/article/view/11985/10730

[15] O. Salazar (2017). "Interfaces adaptativas personalizadas para brindar recomendaciones en repositorios de objetos de aprendizaje2. Revista Tecnura, Vol 21, No 53, pp107-118, doi: 10.14483/22487638.9287. https://revistas.udistrital.edu.co/index.php/Tecnura/article/ view/9287/13447

[16] J. Dodero (2013). ASCETA: Accesibilidad a servicios y contenidos educativos mediante tecnologías del aprendizaje(Accessing educational contents and services with learningtechnologies. XV Simposio Internacional de Tecnologías de la Información y las Comunicaciones en la Educación SINTICE. Facultad de informática, Universidad complutense de Madrid. http://adie.es/static_ adie/SINTICE13-ActasCongreso.pdf

[17] P. Lacasa (2005). "Objetos de aprendizaje y significado Learning objects and meaning". Departamento de Psicopedagogía y Educación Física Universidad de Alcalá. RED. Revista de Educación a Distancia. Año IV. Número monográfico V.18 de Octubre de 2005. https://www. um.es/ead/red/M5/lacasa25.pdf 


\section{Tesis}

[18] H. Contreras (2001). "Procesamiento del Lenguaje Natural basado en una gramática de estilos para el idioma español." (tesis de pregrado). Universidad de los Andes, Facultad de Ingeniería Postgrado en Computación. http://www.saber.ula.ve/bitstream/handle/123456789/15961/ CLEI_2001-a218.pdf?sequence $=1$ \&isAllowed $=y$

[19] P. Alcivar (2007). "Sistema de análisis de patrones de navegación usando minería web”. Tesis pregrado. Guayaquil Ecuador. http://www.dspace.espol.edu.ec/bitstream/123456789/5014/2/7998. pdf

[20] V. López (2005). "Interfaces de usuarios adaptativas basada en modelos y agentes software" Tesis doctoral. Universidad de Castilla, La mancha, España. http://www.dsi.uclm.es/personal/ VictorManuelLopez/mipagina/archivos/thesis.pdf

[21] O. Salazar (2015). "Modelo de Sistema Multi-Agente ubicuo adaptativo y sensible al contexto para ofrecer recomendaciones personalizadas de recursos educativos basado en ontologías". Tesis de Maestría en Ingeniería de Sistemas, Universidad Nacional de Colombia - Sede Medellín 2015. http://bdigital.unal.edu.co/48308/1/1152184079. pdf

[22] Quiroz (2017). Modelo de interfaz adaptativa para recomendación de objetos de aprendizaje basado en perfiles de usuario, agentes inteligentes y ontologías. Universidad Nacional de Colombia Facultad de Minas. Departamento de Ciencias de la Computación y de la Decisión. Medellín, Colombia. https://scielo.conicyt.cl/scielo.php?script=sci_arttext\&pid $=$ S0718-07642018000600295

[23] J. Roldan (2018). "Adaptive and Immersive Interfaces to Improve Situational Awareness in MultiRobot Missions” (Tesis doctoral) Thecnical University of Madrid. Madrid España. Recuperado de http://oa.upm.es/52353/1/JUAN_JESUS_ROLDAN_GOMEZ_B.pdf

[24] J. Sánchez (1999). "Estimación de gramáticas incontextuales probabilísticas y su aplicación en modelización del lenguaje”. Universidad Politécnica de Valencia, Tesis para optar al grado de Doctor en Informática Valencia. https://dialnet.unirioja.es/servlet/tesis?codigo=10225

[25] Serrano, M. (2017). Objetos de Aprendizaje . Instituto Latinoamericano de la Comunicación Educativa Red Escolar. http://red.ilce.edu.mx/sitios/revista/e_formadores_oto_10/articulos/angeles_serrano_nov10.pdf. Consultado 2017. 